\title{
QCD Crossover at Finite Chemical Potential from Lattice Simulations
}

\author{
Szabolcs Borsanyi®, ${ }^{1}$ Zoltan Fodor, ${ }^{1,2,3,4}$ Jana N. Guenther, ${ }^{1,5}$ Ruben Kara $\odot,{ }^{1}$ Sandor D. Katz, ${ }^{2}$ Paolo Parotto®, ${ }^{1}$ \\ Attila Pasztor, ${ }^{2}$ Claudia Ratti, ${ }^{6}$ and Kálman K. Szabó ${ }^{1,3}$ \\ ${ }^{1}$ Department of Physics, Wuppertal University, Gaussstrasse 20, D-42119 Wuppertal, Germany \\ ${ }^{2}$ Institute for Theoretical Physics, ELTE Eötvös Loránd University, Pázmány P. sétány 1/A, H-1117 Budapest, Hungary \\ ${ }^{3}$ Jülich Supercomputing Centre, Forschungszentrum Jülich, D-52425 Jülich, Germany \\ ${ }^{4}$ Physics Department, UCSD, San Diego, California 92093, USA \\ ${ }^{5}$ University of Regensburg, Department of Physics, Regensburg D-93053, Germany \\ ${ }^{6}$ Department of Physics, University of Houston, Houston, Texas 77204, USA
}

(Received 11 February 2020; revised 5 May 2020; accepted 1 July 2020; published 29 July 2020)

\begin{abstract}
We provide the most accurate results for the QCD transition line so far. We optimize the definition of the crossover temperature $T_{c}$, allowing for its very precise determination, and extrapolate from imaginary chemical potential up to real $\mu_{B} \approx 300 \mathrm{MeV}$. The definition of $T_{c}$ adopted in this work is based on the observation that the chiral susceptibility as a function of the condensate is an almost universal curve at zero and imaginary $\mu_{B}$. We obtain the parameters $\kappa_{2}=0.0153(18)$ and $\kappa_{4}=0.00032(67)$ as a continuum extrapolation based on $N_{t}=10,12,16$ lattices with physical quark masses. We also extrapolate the peak value of the chiral susceptibility and the width of the chiral transition along the crossover line. In fact, both of these are consistent with a constant function of $\mu_{B}$. We see no sign of criticality in the explored range.
\end{abstract}

DOI: 10.1103/PhysRevLett.125.052001

Introduction.-One of the most important open problems in the study of quantum chromodynamics (QCD) at finite temperature and density is the determination of the phase diagram of the theory in the temperature $(T)$-baryochemical potential $\left(\mu_{B}\right)$ plane. It is now established by first principle lattice QCD calculations that the transition at $\mu_{B}=0$ is a smooth crossover [1,2] for physical quark masses. Due to the lack of a real phase transition, the crossover temperature is of course ambiguous, since different definitions can lead to different values for it. Observables related to chiral symmetry (i.e., the chiral condensate and its susceptibility) yield a transition temperature around $155-160 \mathrm{MeV}$ [3-6].

Extending our knowledge to the $\mu_{B}>0$ part of the phase diagram turns out to be very challenging due to the notorious sign problem. Since this makes direct simulation at finite $\mu_{B}$ impossible, the state of the art for finite density QCD on fine lattices is to use one of two extrapolation methods. The first method is the direct calculation of Taylor coefficients [7-17] using simulations at $\mu_{B}=0$, while the second is to use simulations at imaginary chemical potentials $\left(\mu_{B}^{2}<0\right)$, where the sign problem is absent, and later perform an extrapolation of different quantities to a real chemical potential $\left(\mu_{B}^{2}>0\right)$ [18-31]. It is often conjectured

Published by the American Physical Society under the terms of the Creative Commons Attribution 4.0 International license. Further distribution of this work must maintain attribution to the author(s) and the published article's title, journal citation, and DOI. Funded by SCOAP ${ }^{3}$. that in the $\left(T, \mu_{B}\right)$ plane the crossover line, departing from $\left(T_{c}, \mu_{B}=0\right)$, eventually turns into a first-order transition line. The point $\left(T_{\mathrm{CEP}}, \mu_{\mathrm{CEP}}\right)$ separating the crossover and the first-order transitions is known as the critical endpoint (CEP), where the transition is expected to be of second order. Though there have been attempts in extracting information about the location of the supposed CEP from lattice simulations [15,26,32-37], these attempts face great difficulties as extrapolation-type methods have the property of giving reliable results mostly in the immediate vicinity of $\mu_{B}=0$.

In this Letter, we address the problem of calculating the Taylor coefficients of the crossover temperature around $\mu_{B}=0$, parameterized as follows:

$$
\frac{T_{c}\left(\mu_{B}\right)}{T_{c}\left(\mu_{B}=0\right)}=1-\kappa_{2}\left[\frac{\mu_{B}}{T_{c}\left(\mu_{B}\right)}\right]^{2}-\kappa_{4}\left[\frac{\mu_{B}}{T_{c}\left(\mu_{B}\right)}\right]^{4} \ldots
$$

along the phenomenologically relevant strangeness neutrality line. In this work we improve the uncertainty on $\kappa_{4}$ available in the literature [16] by a factor of 6, giving a state-of-the-art determination of the crossover line in the $\left(T, \mu_{B}\right)$ plane. In particular, as we will show, at chemical potentials $\mu_{B}>200 \mathrm{MeV}$, the error on the $T_{c}$ extrapolation is dominated by the subleading coefficients, e.g., $\kappa_{4}$. The coefficients $\kappa_{2}$ and $\kappa_{4}$ can be calculated with either one of the standard extrapolation methods. A direct evaluation of the $\mu_{B}$ derivatives from $\mu_{B}=0$ ensembles was used in Refs. [38,39]. The current state of the art using the $\mu_{B}=0$ simulation method is Ref. [16], which includes the first 

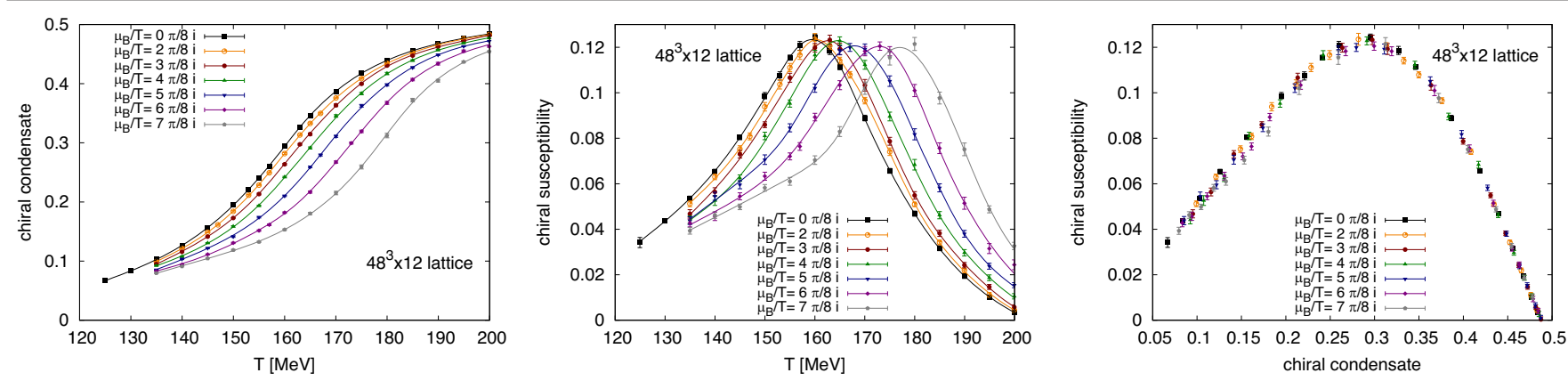

FIG. 1. Renormalized chiral condensate $\langle\bar{\psi} \psi\rangle$ (left) and chiral susceptibility $\chi$ (middle) as functions of the temperature for the intermediate lattice spacing in this study. The black curves correspond to vanishing baryon density, while results for various imaginary values of the chemical potential are shown in other colors. Finally, in the right panel we show the susceptibility as a function of the condensate. In this representation the chemical potential dependence is very weak.

continuum extrapolated results for $\kappa_{4}$. Here we will employ an analytical continuation from imaginary $\mu_{B}$ instead and use lattices as fine as $N_{t}=16$. This is motivated by the fact that the signal-to-noise ratio of higher $\mu_{B}$ derivatives is suppressed with powers of the lattice volume; therefore the calculation of higher order derivatives requires very high statistics. Determinations of $\kappa_{2}$ using the imaginary $\mu_{B}$ method with continuum extrapolation include Refs. [24,25]. Finally, in Ref. [30] the two methods were compared with a careful check of the systematics, and a very good agreement was found for the coefficient $\kappa_{2}$. The transition line was also studied in chiral effective models, see, e.g., the recent Ref. [40].

We also study the strength of the crossover by extrapolating the width of the transition and the value of the chiral susceptibility at the transition to real $\mu_{B}$ in the continuum limit. While one always has to be careful not to overinterpret results from extrapolations, we currently do not see any sign of criticality up to $\mu_{B} \approx 300 \mathrm{MeV}$, as the crossover transition does not get narrower or stronger in this region.

On chiral observables in the transition region.-For the lattice simulations, we use 4-stout improved staggered fermions with an aspect ratio of $L T=4$ and temporal lattice sizes of $N_{t}=10,12,16$. The details of the simulation setup can be found in the Supplemental Material [41]. The use of rooted staggered fermions may come with additional systematic effects that we did not consider in this Letter. Ideally, this work should be repeated with a chiral discretization.

The main observables in this study are the renormalized dimensionless chiral condensate and susceptibility, respectively defined as

$$
\begin{aligned}
\langle\bar{\psi} \psi\rangle & =-\left[\langle\bar{\psi} \psi\rangle_{T}-\langle\bar{\psi} \psi\rangle_{0}\right] \frac{m_{\mathrm{ud}}}{f_{\pi}^{4}}, \\
\chi & =\left[\chi_{T}-\chi_{0}\right] \frac{m_{\mathrm{ud}}^{2}}{f_{\pi}^{4}}, \quad \text { with } \\
\langle\bar{\psi} \psi\rangle_{T, 0} & =\frac{T}{V} \frac{\partial \log Z}{\partial m_{\mathrm{ud}}} \chi_{T, 0}=\frac{T}{V} \frac{\partial^{2} \log Z}{\partial m_{\mathrm{ud}}^{2}},
\end{aligned}
$$

where we assumed isospin symmetry, i.e., $m_{u}=m_{d}=$ $m_{u d}$. In the above equations, the subscripts $T, 0$ indicate values at finite and zero temperature, respectively. In the following, $\langle\bar{\psi} \psi\rangle$ and $\chi$ are always shown after applying the correction to satisfy $n_{s}=0$ with zero statistical error (see the Supplemental Material [41] for details). The peak height of the susceptibility is an indicator for the strength of the transition, while the peak position in temperature serves as a definition for the chiral crossover temperature. It was pointed out in Refs. $[3,4]$ that different normalizations of the susceptibility, such as using $1 / f_{\pi}^{4}$ or $1 / T^{4}$ to define $\chi$ in Eq. (2), can shift the peak position by $11 \mathrm{MeV}$. This difference could be considered as a measure for the broadness of the chiral transition.

Our normalization choice in Eq. (2) was motivated by two observations, shown in Fig. 1 and explained below. These observations (together with the improved statistics and the more accurate tuning of $\mu_{S}\left(\mu_{B}\right)$ to $n_{S}=0$ ) allow a very precise determination of $T_{c}$ as a function of imaginary chemical potential, which in turn allows a precise determination of the parameters $\kappa_{2}$ and $\kappa_{4}$. We explored the chiral condensate and susceptibility in a broad range of imaginary baryochemical potential. In all panels of Fig. 1, the black curves correspond to $\mu_{B}=0$. In the left and middle panel we show the chiral condensate and susceptibility as functions of the temperature. By construction, our renormalized condensate is zero at $T=0$ and positive at high temperature because of the explicit vacuum subtraction and the overall negative sign in Eq. (2). In both panels, one can observe the shifting of the transition toward higher temperatures when an imaginary chemical potential is introduced. In the right panel we show the susceptibility as a function of the condensate. Here we converted the statistical error on the condensate into an additional error on the susceptibility by solving for $\langle\bar{\psi} \psi\rangle(T)=$ const and substituting the resulting $T$ into $\chi(T)$ (also taking the correlation of the statistical errors into account). Our first observation on the right panel of Fig. 1 is that the form of the $\chi(\langle\bar{\psi} \psi\rangle)$ curve is simpler than that of $\chi(T)$ : a low-order (e.g., third or fourth) polynomial can fit the entire transition 
range with an excellent fit quality. The second observation is that there is virtually no chemical potential dependence in the $\chi(\langle\bar{\psi} \psi\rangle)$ function. This way the susceptibility can be modeled as a low-order polynomial of two variables, $\langle\bar{\psi} \psi\rangle$ and $\hat{\mu}=\mu_{B} / T$. Had we used a different normalization for the susceptibility, e.g., $\chi(T) f_{\pi}^{4} / T^{4}$ as we did in Ref. [5], the peak height would be strongly $\mu_{B}$ dependent and the collapse of the $\chi(\langle\bar{\psi} \psi\rangle)$ curves at different (imaginary) chemical potentials would not happen.

The transition line and its analytical continuation.Keeping the previous observations in mind, one can perform a precise determination of $T_{c}$, as defined by the peak of $\chi$ in Eq. (2), for various values of the imaginary chemical potential. $T_{c}\left(\mu_{B}^{2}\right)$ can then be fitted for the coefficients $\kappa_{2}$ and $\kappa_{4}$. This requires the following steps: i) Determine the renormalized condensate $\langle\bar{\psi} \psi\rangle$ and susceptibility $\chi$ in a two-dimensional parameter scan in $T$ and $\operatorname{Im} \mu_{B}$ using lattice simulations. Use these to obtain the susceptibility as a function of the condensate. ii) Search for the peak of $\chi(\langle\bar{\psi} \psi\rangle\rangle)$ through a low-order polynomial fit for each $N_{t}$ and $\operatorname{Im} \mu_{B}$ obtaining $\langle\bar{\psi} \psi\rangle_{c}\left(N_{t}, \operatorname{Im} \mu_{B}\right)$. iii) Use an interpolation of $\langle\bar{\psi} \psi\rangle(T)$ to convert the $\langle\bar{\psi} \psi\rangle_{c}$ to $T_{c}$ for each $\operatorname{Im} \mu_{B} / T$. iv) Perform a global fit of $T_{c}\left(N_{t}, \operatorname{Im} \mu_{B} / T_{c}\right)$ to determine the coefficients $\kappa_{2}$ and $\kappa_{4}$ for $1 / N_{t}^{2}=0$. For this step we use various functions-all containing an independent $\kappa_{6}$-with coefficients depending linearly on $1 / N_{t}^{2}$.

There are ambiguities in steps i)-iv). We estimate their systematics by carrying out many versions of these steps. For all these variations and the estimation of the errors, see the Supplemental Material [41]. We finally obtain

$$
\begin{aligned}
& \kappa_{2}=0.0153 \pm 0.0018 \\
& \kappa_{4}=0.00032 \pm 0.00067 .
\end{aligned}
$$

We stress that the uncertainties on these two quantities are correlated. We put these results in the context of previous lattice studies in Fig. 2. The extrapolated value of $T_{c}\left(\mu_{B}\right)$ is shown in Fig. 3 (green band). Note that the errors on $\kappa_{2}$ and $\kappa_{4}$ are dominated by the statistical errors, as shown in the detailed discussion of the systematic error estimate in the Supplemental Material [41].

Since Ref. [25] we have more than doubled the statistics and introduced a more precise analysis. The overall error on $\kappa_{2}$ has reduced slightly. The main result is the extraction of $\kappa_{4}$. It appears to be a generic feature of deducing Taylor coefficients from polynomial fits: the increased precision on the input data leads to a sensitivity to a higher order coefficient first and only later to a reduction of the error of both coefficients. This feature is also clearly seen in the mock data analysis in the Supplemental Material [41].

In Fig. 3 we also show the comparison to the leading order Taylor expansion result (using only $\kappa_{2}$ ) and the next to leading order result (using $\kappa_{2}$ and $\kappa_{4}$ ). The latter is very close to our full result (for $\mu_{B}<300 \mathrm{MeV}$ ), while the

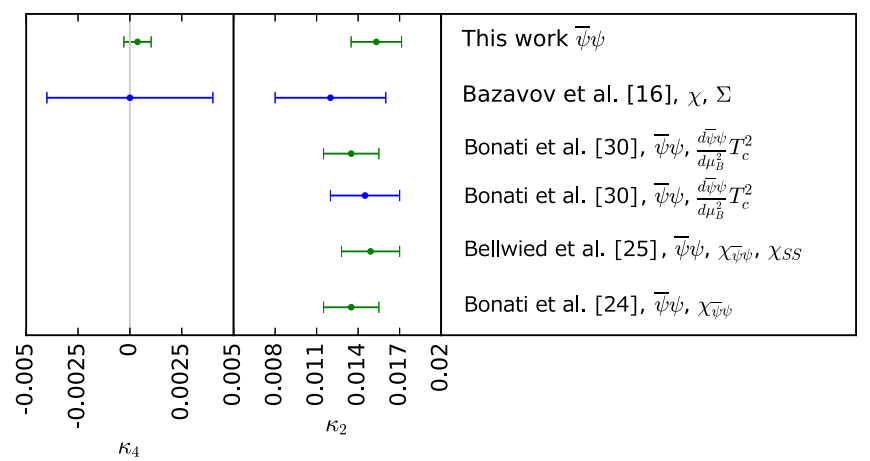

FIG. 2. Compilation of $\kappa_{4}$ (left) and $\kappa_{2}$ (right) coefficients from recent lattice studies. We only include those papers where physical quark masses were used, a controlled continuum extrapolation was performed, and either strangeness neutrality or $\mu_{s}=0$ was considered. [Note that while $\mu_{s}=0$ means $\mu_{S}=\mu_{B} / 3$ for all values of $\mu_{B}$, strangeness neutrality implies $\mu_{S} \approx \mu_{B} / 4$ for small $\mu_{B}$.] The colors encode the numerical approach. Blue points indicate simulations at $\mu_{B}=0$ only, where the $\mu_{B}$ dependence of $T_{c}$ was extracted using a Taylor expansion. If a study used simulations with imaginary chemical potentials in addition, we plot the results with green points, instead. Top data points represent this work, the further references in order: [16,24,25,30].

leading order result has a much smaller uncertainty. Clearly, $\kappa_{2}$ is precise enough. At intermediate $\mu_{B}$, the bottleneck for the precision of $T_{c}\left(\mu_{B}\right)$ is the error on $\kappa_{4}$. We also fitted $\kappa_{6}$, which turned out to be small enough to be irrelevant for $\mu_{B}<300 \mathrm{MeV}$.

In Ref. [25] multiple $T_{c}$ definitions were considered, leading to consistent values for $\kappa_{2}$. However, none of those definitions can match the precision of the $T_{c}$ observable considered here, and precision was a prerequisite for the determination of $\kappa_{4}$.

Extrapolation of the transition width and strength.A natural definition of the width of the susceptibility peak is given by its second derivative at $T_{c}$ as $(\Delta T)^{2}=$ $-\chi\left(T_{c}\right)\left[\left(d^{2} / d T^{2}\right) \chi\right]_{T=T_{c}}^{-1}$. Unfortunately, evaluating this quantity is numerically difficult, so we introduce a simple width parameter $\sigma$ as a proxy for $\Delta T$ via

$$
\langle\bar{\psi} \psi\rangle\left(T_{c} \pm \sigma / 2\right)=\langle\bar{\psi} \psi\rangle_{c} \pm \Delta\langle\bar{\psi} \psi\rangle / 2,
$$

with $\langle\bar{\psi} \gamma \psi\rangle_{c}=0.285$ and $\Delta\langle\bar{\psi} \psi\rangle=0.14$. The choice of the range in $\langle\bar{\psi} \psi\rangle$ is such that it is consistent with a linear behavior within our error bars, meaning that the ratio $\Delta\langle\bar{\psi} \psi\rangle\rangle / \sigma$ can be used as a proxy for $\left.(d / d T)\langle\bar{\psi} \psi\rangle\right|_{T=T_{c}}$ as well. The exact range in $\langle\bar{\psi} \psi\rangle$ is chosen such that $\sigma$ coincides with $\Delta T$ at zero and imaginary $\mu_{B}$. A more detailed discussion of the width parameter can be found in the Supplemental Material [41].

We conclude that the half-width of the transitionshown in the upper panel of Fig. 4-is consistent with a constant up to $\mu_{B} \approx 300 \mathrm{MeV}$ within the uncertainty from 


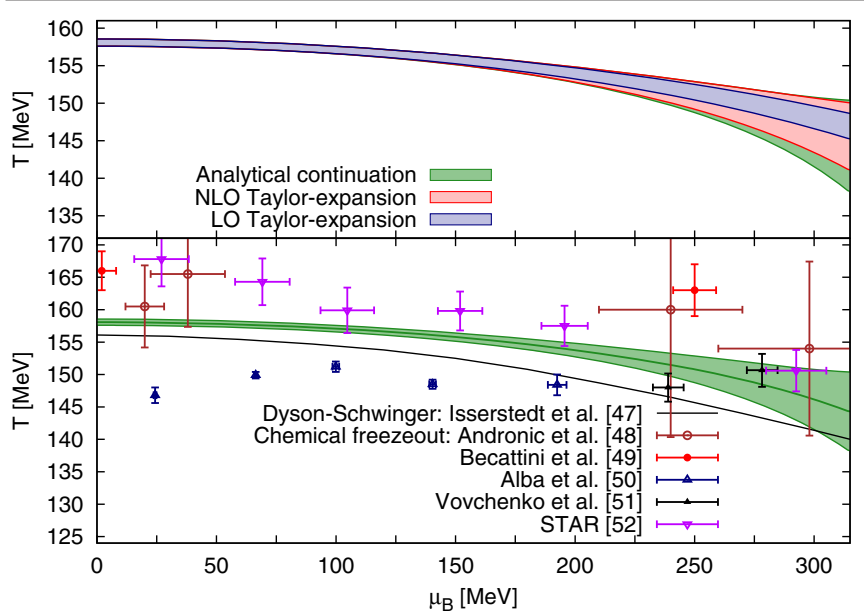

FIG. 3. Top: Transition line extrapolated from lattice simulations at imaginary chemical potential using an analytical continuation with the ansatz used in step iv) of our analysis (green band) compared with an extrapolation using the formula in Eq. (1) up to the order of $\kappa_{4}$ (red band) or up to $\kappa_{2}$ (blue band). The proximity of the full and NLO result suggests that the higher order corrections are small in the range of $\mu_{B}$ considered here. Note that considering only the error bar of $\kappa_{2}$ underestimates the full error. The numerical values for the final analytical continuation, together with its error, are tabulated in the Supplemental Material [41]. Bottom: Crossover line from the lattice compared with a prediction from truncated Dyson-Schwinger equations [47] and some estimates of the chemical freeze-out parameters in heavy ion collisions [48-52]. Note that the width of the green band is not a representation of the width of the crossover region; it depicts the statistical and systematic errors achievable with the particular definition of the crossover temperature $T_{c}$ adopted in this work. Note also that the definition of the crossover temperature adopted in Ref. [47] is different from the one used in this work.

the extrapolation (we note that $50 \%$ uncertainty is reached at $\mu_{B} \approx 280 \mathrm{MeV}$ ).

Finally, as a proxy for the strength of the crossover, we study the value of the chiral susceptibility at the crossover temperature. We get this for each $\operatorname{Im} \mu_{B}$ and $N_{t}$ as a byproduct of steps i)-ii) of the analysis for $\kappa_{2}$ and $\kappa_{4}$. If one then performs a continuum extrapolation of the resulting values for fixed values of $\operatorname{Im} \mu_{B}$, one gets the lower panel of Fig. 4. Again, we see a very mild $\hat{\mu}_{B}^{2}$ dependence, consistent with a constant.

Summary and discussion.-The main result of this work is a precise determination of the parameters $\kappa_{2}$ and $\kappa_{4}$ of the crossover line in finite density QCD. For the determination of the crossover line, we used the experimentally relevant $\mu_{S}\left(\mu_{B}\right)$ tuned to keep $n_{S}=0$. Based on the observation that the chiral susceptibility as a function of the condensate is a rather simple function, only weakly dependent on the imaginary chemical potential, we were able to obtain the transition temperature as a function of the imaginary chemical potential to very high accuracy. These pure lattice results can be used for further model building and are
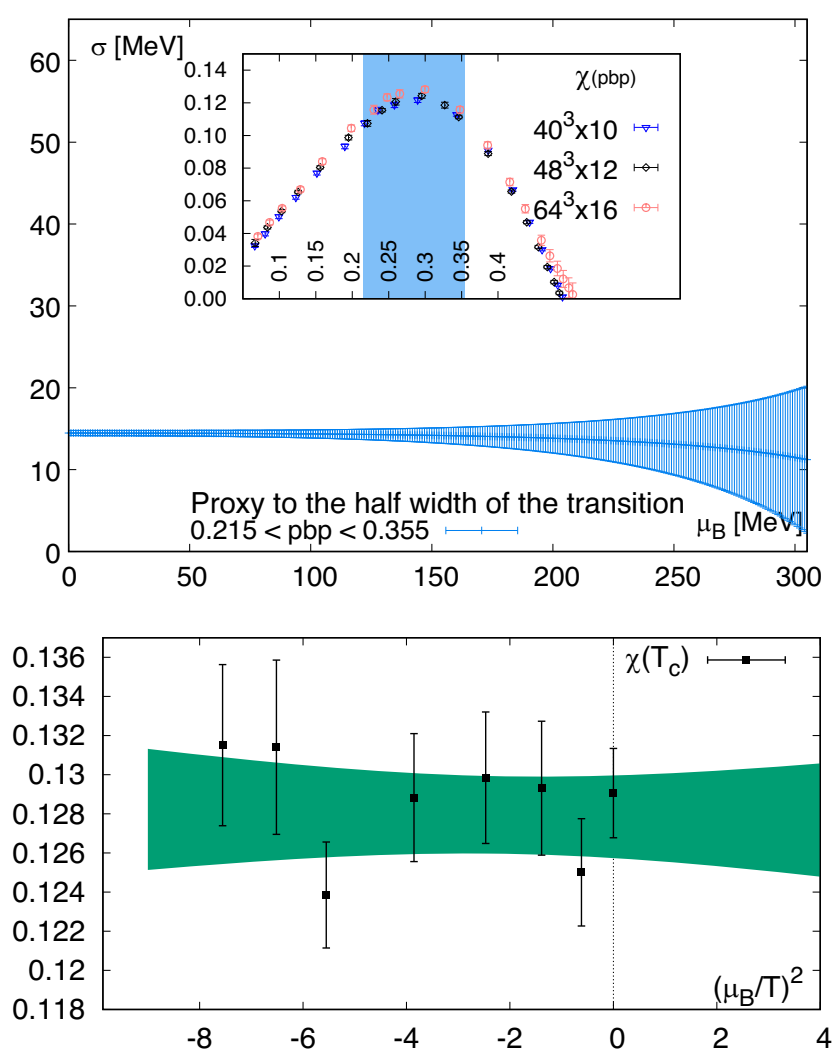

FIG. 4. Top: Half-width $\sigma$ of the transition defined in Eq. (4) using the temperature difference of the contours $\langle\bar{\psi} \psi\rangle=0.355$ and $\langle\bar{\psi} \psi\rangle=0.215$. In the insert we show a plot of the $\chi(\langle\bar{\psi} \psi\rangle)$ peak, where the shaded region corresponds to $\langle\bar{\psi} \psi\rangle_{c} \pm \Delta\langle\bar{\psi} \psi\rangle / 2$. Both are extrapolated to real $\mu_{B}$. Bottom: Result of a $\mu_{B}$ by $\mu_{B}$ analysis for the value of the chiral susceptibility at the crossover temperature after continuum extrapolation and including the systematic errors for $L T_{c}=4$. The green band shows a linear extrapolation in $\hat{\mu}_{B}^{2}$.

summarized in the Supplemental Material [41]. The high precision data at imaginary $\mu_{B}$ in turn allowed us to fit the $\mu_{B}^{2}$ and $\mu_{B}^{4}$ Taylor coefficients of the crossover temperature $\kappa_{2}$ and $\kappa_{4}$. In particular, while our determination of $\kappa_{4}$ is still consistent with zero, the error is 6 times smaller than the one previously available in the literature and therefore represents the state of the art in the study of the phase diagram in the $\left(T, \mu_{B}\right)$ plane with current lattice techniques. As a byproduct, we also obtain the most precise value for the central temperature of the crossover at $\mu_{B}=0$ so far, as well as the width of the transition:

$$
\begin{aligned}
T_{c}\left(L T=4, \mu_{B}=0\right) & =158.0 \pm 0.6 \mathrm{MeV} \\
\Delta T\left(L T=4, \mu_{B}=0\right) & =15 \pm 1 \mathrm{MeV}
\end{aligned}
$$

We briefly discuss the meaning of these small errors in the Supplemental Material [41].

We also studied the strength of the phase transition as a function of $\mu_{B}$ by extrapolating our proxy for the transition 
width and the peak of the chiral susceptibility from imaginary chemical potentials. Even though one has to be careful with extrapolations, we see no sign of the transition getting stronger up to $\mu_{B} \approx 300 \mathrm{MeV}$.

This project was funded by the DFG Grant No. SFB/ TR55. The project also received support from the BMBF Grant No. 05P18PXFCA. This work was also supported by the Hungarian National Research, Development, and Innovation Office, NKFIH Grant Nos. KKP126769 and K113034. A. P. is supported by the J. Bolyai Research Scholarship of the Hungarian Academy of Sciences and by the UNKP-19-4 New National Excellence Program of the Ministry for Innovation and Technology. This material is based upon work supported by the National Science Foundation under Grant No. PHY-1654219 and by the U.S. Department of Energy, Office of Science, Office of Nuclear Physics within the framework of the Beam Energy Scan Topical (BEST) Collaboration. The authors gratefully acknowledge the Gauss Centre for Supercomputing e.V. (www.gauss-centre.eu) for funding this project by providing computing time on the GCS Supercomputer JURECA/Booster at Jülich Supercomputing Centre (JSC), on HAZELHEN at HLRS, Stuttgart as well as on SUPERMUC-NG at LRZ, Munich. We acknowledge PRACE for awarding us access to Piz Daint hosted at CSCS, Switzerland. C. R. also acknowledges the support from the Center of Advanced Computing and Data Systems at the University of Houston.

[1] Y. Aoki, G. Endrodi, Z. Fodor, S. Katz, and K. Szabo, Nature (London) 443, 675 (2006).

[2] T. Bhattacharya, M. I. Buchoff, N. H. Christ, H.-T. Ding, R. Gupta, C. Jung, F. Karsch, Z. Lin, R. Mawhinney, G. McGlynn et al., Phys. Rev. Lett. 113, 082001 (2014).

[3] Y. Aoki, Z. Fodor, S. Katz, and K. Szabo, Phys. Lett. B 643, 46 (2006).

[4] Y. Aoki, S. Borsanyi, S. Durr, Z. Fodor, S. D. Katz, S. Krieg, and K. Szabo, J. High Energy Phys. 06 (2009) 088.

[5] S. Borsanyi, Z. Fodor, C. Hoelbling, S. D. Katz, S. Krieg, C. Ratti, and K. K. Szabó (Wuppertal-Budapest Collaboration), J. High Energy Phys. 09 (2010) 073.

[6] A. Bazavov, T. Bhattacharya, M. Cheng, C. DeTar, H. Ding et al., Phys. Rev. D 85, 054503 (2012).

[7] C. Allton, S. Ejiri, S. Hands, O. Kaczmarek, F. Karsch, E. Laermann, C. Schmidt, and L. Scorzato, Phys. Rev. D 66, 074507 (2002).

[8] C. Allton, M. Doring, S. Ejiri, S. Hands, O. Kaczmarek, F. Karsch, E. Laermann, and K. Redlich, Phys. Rev. D 71, 054508 (2005).

[9] R. V. Gavai and S. Gupta, Phys. Rev. D 78, 114503 (2008).

[10] S. Basak et al. (MILC Collaboration), Proc. Sci., LATTICE2008 (2008) 171 [arXiv:0910.0276].

[11] S. Borsanyi, Z. Fodor, S. D. Katz, S. Krieg, C. Ratti, and K. Szabó, J. High Energy Phys. 01 (2012) 138.
[12] S. Borsanyi, G. Endrodi, Z. Fodor, S. Katz, S. Krieg, C. Ratti, and K. K. Szabó, J. High Energy Phys. 08 (2012) 053.

[13] R. Bellwied, S. Borsanyi, Z. Fodor, S. D. Katz, A. Pasztor, C. Ratti, and K. K. Szabo, Phys. Rev. D 92, 114505 (2015).

[14] H. T. Ding, S. Mukherjee, H. Ohno, P. Petreczky, and H. P. Schadler, Phys. Rev. D 92, 074043 (2015).

[15] A. Bazavov et al., Phys. Rev. D 95, 054504 (2017).

[16] A. Bazavov et al., Phys. Lett. B 795, 15 (2019).

[17] A. Bazavov et al., Phys. Rev. D 101, 074502 (2020).

[18] P. de Forcrand and O. Philipsen, Nucl. Phys. B642, 290 (2002).

[19] M. D'Elia and M.-P. Lombardo, Phys. Rev. D 67, 014505 (2003).

[20] M. D'Elia and F. Sanfilippo, Phys. Rev. D 80, 014502 (2009).

[21] P. Cea, L. Cosmai, and A. Papa, Phys. Rev. D 89, 074512 (2014).

[22] C. Bonati, P. de Forcrand, M. D’Elia, O. Philipsen, and F. Sanfilippo, Phys. Rev. D 90, 074030 (2014).

[23] P. Cea, L. Cosmai, and A. Papa, Phys. Rev. D 93, 014507 (2016).

[24] C. Bonati, M. D’Elia, M. Mariti, M. Mesiti, F. Negro, and F. Sanfilippo, Phys. Rev. D 92, 054503 (2015).

[25] R. Bellwied, S. Borsanyi, Z. Fodor, J. Günther, S. D. Katz, C. Ratti, and K. K. Szabo, Phys. Lett. B 751, 559 (2015).

[26] M. D'Elia, G. Gagliardi, and F. Sanfilippo, Phys. Rev. D 95, 094503 (2017).

[27] J. Gunther, R. Bellwied, S. Borsanyi, Z. Fodor, S. D. Katz, A. Pasztor, and C. Ratti, EPJ Web Conf. 137, 07008 (2017); Nucl. Phys. A967, 720 (2017).

[28] P. Alba et al., Phys. Rev. D 96, 034517 (2017).

[29] V. Vovchenko, A. Pasztor, Z. Fodor, S. D. Katz, and H. Stoecker, Phys. Lett. B 775, 71 (2017).

[30] C. Bonati, M. D’Elia, F. Negro, F. Sanfilippo, and K. Zambello, Phys. Rev. D 98, 054510 (2018).

[31] S. Borsanyi, Z. Fodor, J. N. Guenther, S. K. Katz, K. K. Szabo, A. Pasztor, I. Portillo, and C. Ratti, J. High Energy Phys. 10 (2018) 205.

[32] Z. Fodor and S. Katz, J. High Energy Phys. 03 (2002) 014.

[33] Z. Fodor and S. Katz, J. High Energy Phys. 04 (2004) 050.

[34] Z. Fodor, M. Giordano, J. N. Günther, K. Kapás, S. D. Katz, A. Pásztor, I. Portillo, C. Ratti, D. Sexty, and K. K. Szabó, Nucl. Phys. A982, 843 (2019).

[35] M. Giordano and A. Pasztor, Phys. Rev. D 99, 114510 (2019).

[36] S. Mukherjee and V. Skokov, arXiv:1909.04639.

[37] M. Giordano, K. Kapas, S. D. Katz, D. Nogradi, and A. Pasztor, Phys. Rev. D 101, 074511 (2020).

[38] O. Kaczmarek, F. Karsch, E. Laermann, C. Miao, S. Mukherjee, P. Petreczky, C. Schmidt, W. Soeldner, and W. Unger, Phys. Rev. D 83, 014504 (2011).

[39] G. Endrodi, Z. Fodor, S. Katz, and K. Szabo, J. High Energy Phys. 04 (2011) 001.

[40] K. Zarembo, Pis'ma Zh. Eksp. Teor. Fiz. 110, 147 (2019) [JETP Lett. 110, 155 (2019)].

[41] See Supplemental Material at http://link.aps.org/ supplemental/10.1103/PhysRevLett.125.052001 for the technical details of this study, which includes Refs. [42-46]. 
[42] M. Tanabashi et al. (Particle Data Group), Phys. Rev. D 98, 030001 (2018).

[43] S. Borsanyi, S. Durr, Z. Fodor, C. Hoelbling, S. D. Katz et al., J. High Energy Phys. 09 (2012) 010.

[44] S. Borsanyi et al., Nature (London) 539, 69 (2016).

[45] R. Bellwied, S. Borsanyi, Z. Fodor, J. N. Guenther, J. Noronha-Hostler, P. Parotto, A. Pasztor, C. Ratti, and J. M. Stafford, Phys. Rev. D 101, 034506 (2020).

[46] C. Bonati, M. D’Elia, M. Mariti, M. Mesiti, F. Negro, and F. Sanfilippo, Phys. Rev. D 90, 114025 (2014).

[47] P. Isserstedt, M. Buballa, C. S. Fischer, and P. J. Gunkel, Phys. Rev. D 100, 074011 (2019).
[48] A. Andronic, P. Braun-Munzinger, and J. Stachel, Nucl. Phys. A772, 167 (2006).

[49] F. Becattini, M. Bleicher, T. Kollegger, T. Schuster, J. Steinheimer, and R. Stock, Phys. Rev. Lett. 111, 082302 (2013).

[50] P. Alba, W. Alberico, R. Bellwied, M. Bluhm, V. Mantovani Sarti, M. Nahrgang, and C. Ratti, Phys. Lett. B 738, 305 (2014).

[51] V. Vovchenko, V. V. Begun, and M. I. Gorenstein, Phys. Rev. C 93, 064906 (2016).

[52] L. Adamczyk et al. (STAR Collaboration), Phys. Rev. C 96, 044904 (2017). 